\title{
A Dominant-Negative GFI1B Mutation in the Gray Platelet Syndrome
}

\author{
Davide Monteferrario, M.S., Nikhita A. Bolar, M.S., Anna E. Marneth, M.S., \\ Konnie M. Hebeda, M.D., Ph.D., Saskia M. Bergevoet, B.S., Hans Veenstra, B.S., \\ Britta A.P. Laros-van Gorkom, M.D., Ph.D., Marius A. MacKenzie, M.D., Ph.D., \\ Cyrus Khandanpour, M.D., Ph.D., Lacramiora Botezatu, Ph.D., \\ Erik Fransen, Ph.D., Guy Van Camp, Ph.D., Anthonie L. Duijnhouwer, M.D., \\ Simone Salemink, M.S., Brigith Willemsen, B.S., Gerwin Huls, M.D., Ph.D., \\ Frank Preijers, Ph.D., Waander Van Heerde, Ph.D., Joop H. Jansen, Ph.D., \\ Marlies J.E. Kempers, M.D., Ph.D., Bart L. Loeys, M.D., Ph.D., \\ Lut Van Laer, Ph.D., and Bert A. Van der Reijden, Ph.D.
}

\section{SUMMARY}

The gray platelet syndrome is a hereditary, usually autosomal recessive bleeding disorder caused by a deficiency of alpha granules in platelets. We detected a nonsense mutation in the gene encoding the transcription factor GFI1B (growth factor independent 1B) that causes autosomal dominant gray platelet syndrome. Both gray platelets and megakaryocytes had abnormal marker expression. In addition, the megakaryocytes had dysplastic features, and they were abnormally distributed in the bone marrow. The GFI1B mutant protein inhibited nonmutant GFI1B transcriptional activity in a dominant-negative manner. Our studies show that GFI1B, in addition to being causally related to the gray platelet syndrome, is key to megakaryocyte and platelet development.

P LATELETS ARE FORMED THROUGH FRAGMENTATION OF MEGAKARYOcytes that reside in the bone marrow. ${ }^{1,2}$ Platelet alpha granules, which are by far the most abundant platelet organelles, store proteins that stimulate platelet adhesiveness, hemostasis, and wound healing. ${ }^{3,4}$ The gray platelet syndrome is an inherited bleeding disorder characterized by defective production of alpha granules. ${ }^{5,6}$ Patients with this syndrome have reduced numbers of larger-than-normal platelets, and on light microscopy these platelets have a typical gray appearance caused by the lack of alpha granules. For a final diagnosis, the lack of alpha granules must be confirmed by means of electron microscopy. ${ }^{7}$ Clinically, patients with the gray platelet syndrome have bleeding tendencies with variable severity. ${ }^{8}$ Additional features associated with this syndrome are bone marrow fibrosis and the capture of neutrophils within megakaryocytes in a process known as emperipolesis (the presence of an intact cell within the cytoplasm of another intact cell). ${ }^{6,8}$

It has been proposed that the alpha-granule deficiency in the gray platelet syndrome can be attributed to the failure of megakaryocytes to efficiently route endogenous proteins into alpha granules, thereby hampering the maturation of these granules. ${ }^{9,10}$ Although the gray platelet syndrome is most often an autosomal recessive disease, families with an autosomal dominant inheritance pattern have been described as well. ${ }^{6,11}$ Recently, biallelic NBEAL2 missense mutations have been
From the Department of Laboratory Medicine, Laboratory of Hematology, Radboud University Medical Center, Nijmegen Center for Molecular Life Sciences (D.M., A.E.M., S.M.B., H.V., G.H., F.P., W.V.H., J.H.J., B.A.V.R.) and the Departments of Pathology (K.M.H., B.W.), Hematology (B.A.P.L.G., M.A.M., G.H.), Cardiology (A.L.D.), and Human Genetics (S.S., M.J.E.K.), Radboud University Medical Center - all in Nijmegen, the Netherlands; the Department of Medical Genetics, Antwerp University Hospital and University of Antwerp (N.A.B., E.F., G.V.C., B.L.L., L.V.L.) and the Statua Center for Statistics, University of Antwerp (E.F.), Antwerp, Belgium; and the Department of Hematology, University Hospital, Essen, Germany (C.K., L.B.). Address reprint requests to Dr. Van der Reijden at Radboud University Medical Center, Department of Laboratory Medicine, Huispost 475, Geert Grooteplein zuid 8, 6525 GA Nijmegen, the Netherlands, or at bert.vanderreijden@ radboudumc.nl; or to Dr. Van Laer at the University of Antwerp, Department of Medical Genetics, Prins Boudewijnlaan 43, B-2650 Edegem, Belgium, or at lut vanlaer@uantwerp.be.

Mr. Monteferrario, Ms. Bolar, and Ms. Marneth contributed equally to this article.

This article was published on December 10 , 2013, and updated on August 20, 2015, at NEJM.org.

N Engl J Med 2014;370:245-53. DOI: 10.1056/NEJMoal308130

Copyright () 2013 Massachusetts Medical Society. 
identified in an autosomal recessive form of the gray platelet syndrome. ${ }^{12-14}$ NBEAL2 localizes to the dense tubular system in platelets, ${ }^{12}$ but how NBEAL2 mutations contribute to defective alphagranule formation remains unknown. No genes have been implicated in autosomal dominant gray platelet syndrome. Here we report on the identification of a nonsense mutation in the transcription-factor gene, growth factor independent $1 \mathrm{~B}$ (GFI1B), in a family with autosomal dominant gray platelet syndrome. GFI1B and its paralog GFI1 function as transcriptional repressors and play fundamental roles during hematopoiesis. ${ }^{15-20}$ Both proteins contain a highly conserved $\mathrm{C}$-terminal region that includes three zinc fingers that are necessary for interaction with DNA and an identical $\mathrm{N}$-terminal region through which epigenetic modifiers required to inhibit transcription are recruited. ${ }^{16,21-23}$ In addition, GFI1 and GFI1B interact directly with other transcription factors to coregulate gene expression during blood-cell development. ${ }^{15}$

\section{METHODS}

\section{STUDY FAMILY}

We studied a large family with an autosomal dominant form of thrombopathic thrombocytopenia characterized by mild-to-severe bleeding complications, a diagnosis originally described in 1968. ${ }^{24}$ The thrombopathic disorder in this family consisted of a low platelet count, an increased platelet size, and few alpha granules. An updated evaluation of affected family members according to the current disease classification allowed us to redefine the clinical diagnosis as the gray platelet syndrome (number 139090 in the Online Mendelian Inheritance in Man database).

The study was approved by the institutional review board of Radboud University Medical Center. Written informed consent was obtained from all family members who underwent evaluation.

\section{LABORATORY ANALYSES}

The bleeding score was calculated, and morphologic and immunohistochemical studies, electron microscopy, flow cytometry, GFI1B sequencing, and platelet-related analyses were performed according to standard procedures (see the Supplementary Appendix, available with the full text of
Figure 1 (facing page). Characteristics of Autosomal Dominant Gray Platelet Syndrome.

May-Grünwald-Giemsa staining in Panel A shows normal platelets (arrows) obtained from unaffected Family Member II.4. May-Grünwald-Giemsa staining in Panel B shows large gray platelets (arrows) obtained from affected Family Member II.2. Electron-microscopic analysis in Panel $C$ shows a normal platelet with numerous alpha granules (arrows) in a specimen obtained from unaffected Family Member II.4. Electron-microscopic analysis shows gray platelets with few alpha granules, which are poorly developed (arrows), in a specimen obtained from Family Member III.2, and no alpha granules in a specimen obtained from Family Member II.2 (Panels D and E, respectively). Panels F through I show abnormalities in a bone marrow-biopsy specimen obtained from affected Family Member III.2. Laguesse staining in Panel $\mathrm{F}$ reveals stage I bone marrow fibrosis, consisting of a focal network of fine, dark-staining reticulin fibers that extend from the sinuses. Hematoxylin and eosin staining in Panel G shows megakaryocytes that are both larger and smaller than normal, with shapes that deviate from the normal round form (arrows). Some nuclei are irregular and hyperchromatic. Periodic acidSchiff staining in Panel $\mathrm{H}$ shows the capture of neutrophils (arrows) by megakaryocytes (emperipolesis). $\beta_{3}$ integrin-CD61 staining in Panel I shows stretched megakaryocytes abnormally positioned in tandem along the sinuses (arrows).

this article at NEJM.org). Measurements of plasma glycocalicin (Cusabio) and megakaryocyte colony-forming assays (StemCell Technologies) were performed according to the manufacturers' instructions. Linkage analysis based on genomewide single-nucleotide polymorphism array with the use of Linkage 24 DNA Analysis BeadChips (Illumina), GFI1 and GFI1B gene-reporter assays, the method of retroviral transduction, and primary cell cultures are described in the Supplementary Appendix.

\section{RESULTS}

\section{CLINICAL FINDINGS AND LABORATORY ANALYSES}

Morphologic analysis of blood smears from the six affected family members who were evaluated showed a ghostlike, gray appearance of enlarged platelets, in contrast to the normal platelets observed in the two unaffected family members (Fig. 1A and 1B). Electron-microscopic analysis confirmed a marked reduction in the number of alpha granules (Fig. 1C, 1D, and 1E).

Affected family members had moderate-to- 


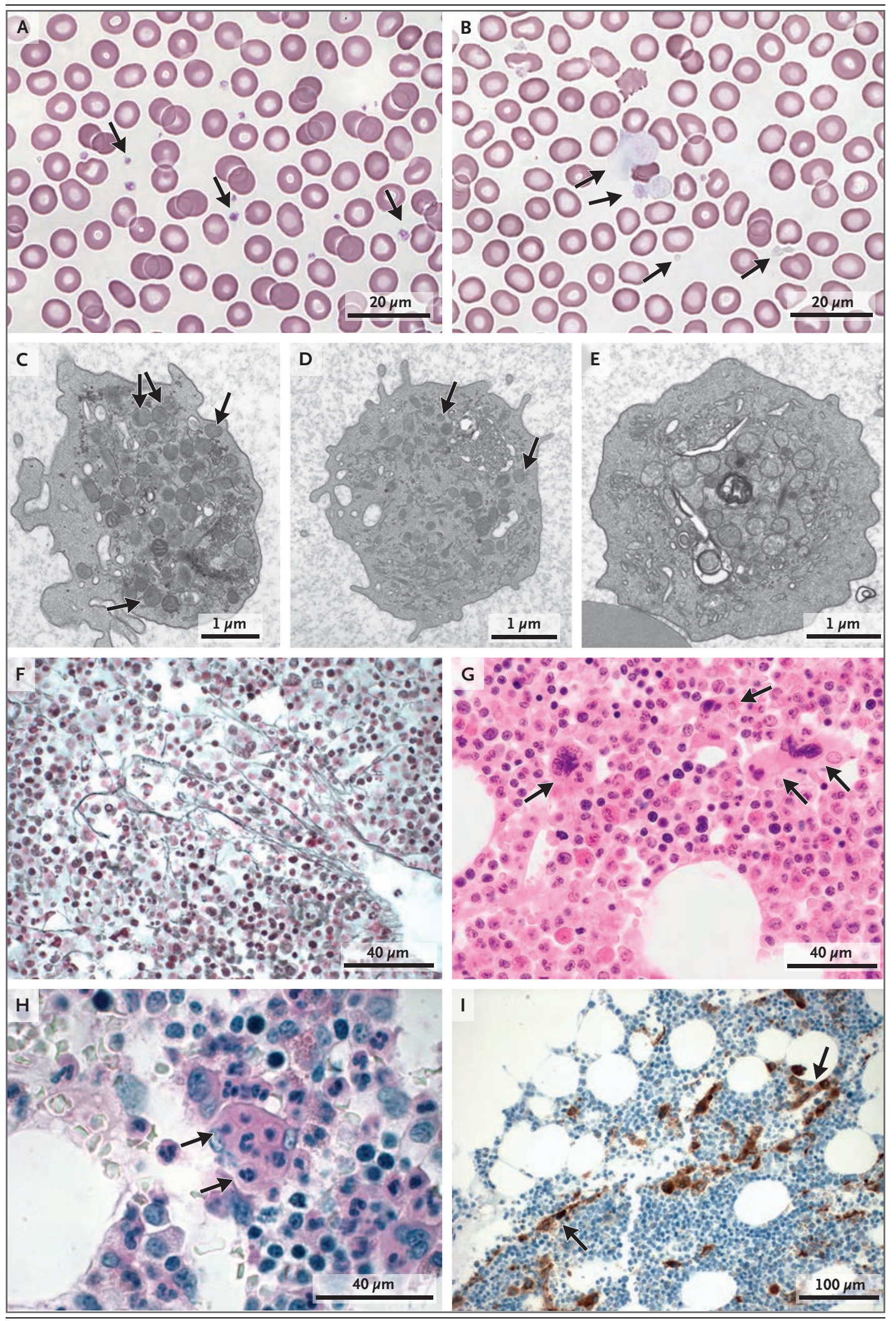


severe bleeding tendencies (Table S1 in the Supplementary Appendix). In addition to reduced platelet counts, diminished platelet factor 4 and $\beta$-thromboglobulin levels were observed in platelets obtained from the affected family members (Table S1 in the Supplementary Appendix).

\section{HISTOPATHOLOGICAL FINDINGS}

Histopathological examination of a bone marrow biopsy specimen obtained from Family Member III.2 (Fig. 2A) showed stage I myelofibrosis (Fig. $1 \mathrm{~F})$ and cellular marrow with an increased number of megakaryocytes that were pleomorphic in size and shape (Fig. 1G, and Fig. S1 in the Supplementary Appendix). Emperipolesis was frequently observed (Fig. 1H, and Fig. S1 in the Supplementary Appendix). Megakaryocytes were clustered along bone marrow sinuses and had stretched features (Fig. 1I, and Fig. S1 in the Supplementary Appendix).

\section{LINKAGE AND SEQUENCE ANALYSES}

To determine the disease-causing mutation, we first performed linkage analyses in 14 family members and identified a candidate locus on chromosome 9q34 with a maximum LOD score of 3.9 (Fig. S2 and S3 in the Supplementary Appendix). Within the linked region, we considered GFI1B to be an excellent candidate gene because it encodes a transcriptional repressor that has been implicated in megakaryopoiesis. ${ }^{17-19}$

Sequence analysis detected a nonsense mutation in exon 6 (c.859C $\rightarrow \mathrm{T}$, p.Gln287^) of GFI1B that completely cosegregated with the gray platelet syndrome (Fig. 2A and 2B, and Fig. S4 and Table S2 in the Supplementary Appendix). The mutation introduces a premature stop codon that is predicted to lead to the formation of a truncated protein $\left(\mathrm{GFI} 1 \mathrm{~B}^{\mathrm{Tr}}\right)$ that lacks 44 carboxyterminal amino acids (Fig. 2B). Since premature stop codons can induce nonsense-mediated messenger RNA (mRNA) decay, we determined whether mutated GFI1B was expressed at the mRNA level. Sequencing of complementary DNA generated from $\mathrm{CD} 34+$ progenitor cells obtained from two affected family members showed that mutated and nonmutated GFIB transcripts were expressed, indicating that the mutated transcript is not targeted for decay (Fig. S4 in the Supplementary Appendix).
Figure 2 (facing page). GFI1B Nonsense Mutation in the Gray Platelet Syndrome, Inhibiting the Function of Nonmutant GFIIB.

Panel A shows the pedigree of the family with the gray platelet syndrome. Squares denote male family members, circles female family members, solid symbols affected family members, open symbols unaffected family members, and slashes deceased family members. Mutation status is indicated below the number of each family member, with $-/$ - indicating the absence and $+/$ - the presence of the GFI1B c.859C $\rightarrow T$, p.G $\ln 287 *$ mutation. Panel B (top) shows that human GFIlB (hGFIlB) contains an N-terminal Snail/Gfi-l (SNAG) domain through which epigenetic modifiers are recruited. At the C-terminal, GFIlB contains six zinc fingers, of which three through five are essential for DNA binding. The GFI1B nonsense mutation (c.859C $\rightarrow \mathrm{T}$, p.G $\ln 287 *$ ) is located in zinc finger 5 (arrow). The resulting truncated protein, GFIlB ${ }^{\mathrm{Tr}}$, lacks four DNAinteracting amino acids in zinc finger 5 (bottom, arrows). GFIIB zinc finger 5 is identical to zinc finger 5 of human, rat, and mouse GFIl ( $\mathrm{h} / \mathrm{r} / \mathrm{mGFIl})$. Panel $\mathrm{C}$ shows that expression of GFII and GFIIB in HEK293T cells results in 60 to $75 \%$ repression of the reporter construct; GFIIB ${ }^{T}$ does not repress the reporter. In cotransfection experiments, GFIlB ${ }^{\text {Tr }}$ inhibited the repression mediated by GFIIB. T bars represent standard errors. May-GrünwaldGiemsa staining in Panel D shows abnormal megakaryocytes (right) characterized by dysplastic features, including hypolobulation of the nucleus and multiple separated nuclei. These abnormal megakaryocytes developed after retroviral expression of $\mathrm{GFIlB}^{\mathrm{Tr}}$ in $\mathrm{mu}$ rine lineage c-kit-positive progenitor cells, followed by megakaryocytic differentiation. Megakaryocytes derived from control vector-transduced bone marrow cells (left) had normal morphologic features.

\section{MOLECULAR AND FUNCTIONAL CHARACTERIZATION OF MUTATED GFIIB}

GFI1B functions as a transcriptional repressor. The truncating mutation is located within zinc finger 5, which is required for DNA binding. This zinc finger is $100 \%$ identical between humans and rodents (rats and mice), and it is identical to zinc finger 5 of the paralog GFI1 (Fig. 2B). ${ }^{20}$ Murine Gfi1 and Gfi1b bind the same DNA consensus sequence, and Gfi1 zinc finger 5 directly interacts with the major groove of the DNA core sequence AATC through four amino acids. ${ }^{22}$ Because the truncated protein GFI1B ${ }^{\text {Tr }}$ lacks all these DNA-interacting amino acids (Fig. 2B), we hypothesized that it would be unable to repress gene expression. To test this hypothesis, we introduced the truncating mutation in a GFI1B expression vector and performed transcriptional 


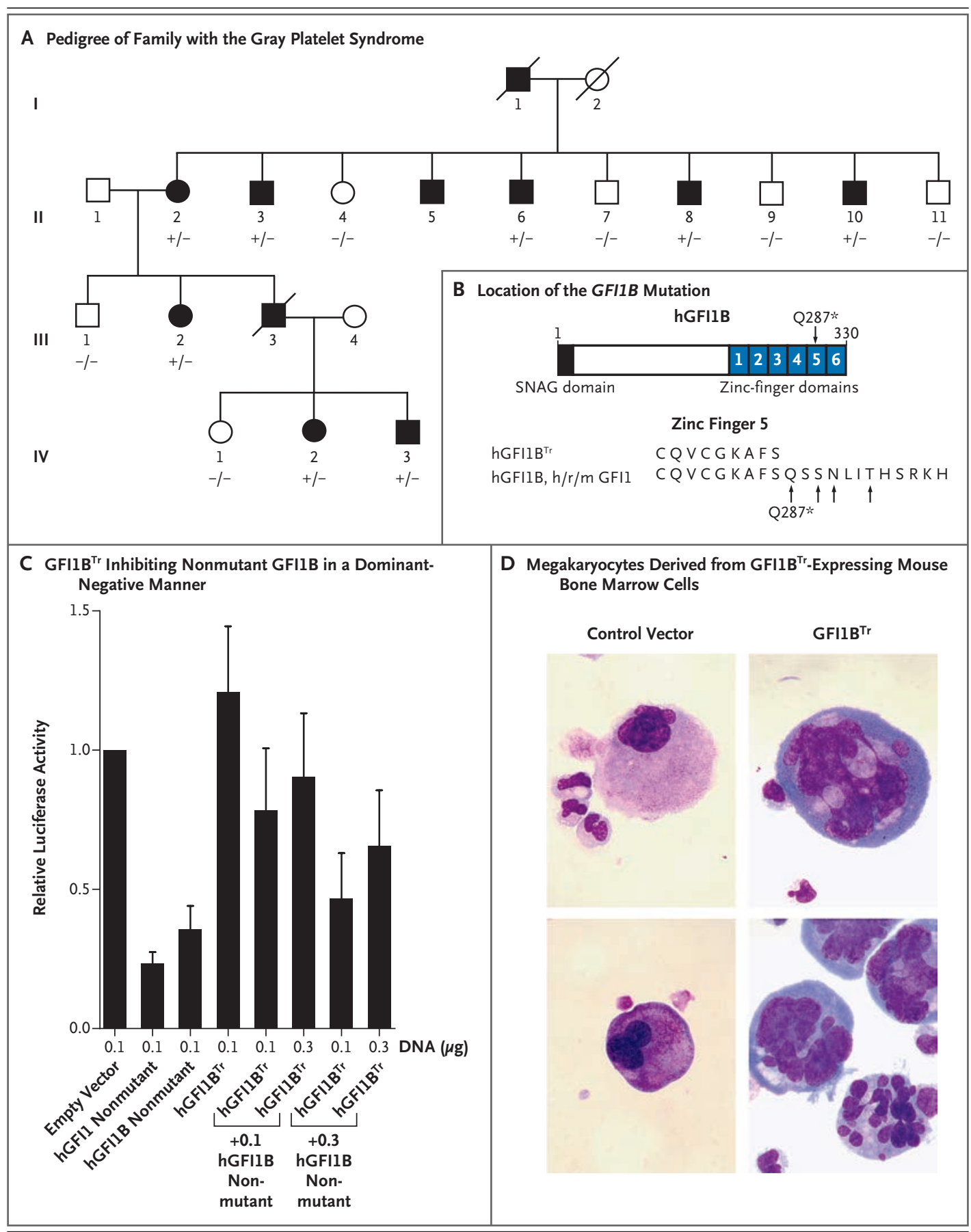

repression assays using the Gfi1 promoter as a When coexpressed, GFI1B ${ }^{\mathrm{Tr}}$ inhibited repression validated Gfi1B target. ${ }^{25}$ In line with the lack of mediated by nonmutant GFI1B, indicating that an intact DNA-binding zinc finger, we observed the mutant interferes with nonmutant GFI1B in a that GFI1B ${ }^{\text {Tr }}$ did not repress the Gfi1 promoter, dominant-negative fashion (Fig. 2C).

whereas the nonmutant GFI1 and GFI1B did (Fig. To validate that GFI1B ${ }^{\text {Tr }}$ adversely affects nor2C, and Fig. S5 in the Supplementary Appendix). mal GFI1B, we expressed GFI1B ${ }^{\mathrm{Tr}}$ in mouse bone 
marrow cells and then induced megakaryocytic differentiation. GFI1B ${ }^{\mathrm{Tr}}$-positive megakaryocytes had several dysplastic features, including hypolobulation of the nuclei, irregular contours, and multiple separated nuclei; these features were not observed in control cells (Fig. 2D, and Fig. S6 in the Supplementary Appendix). These abnormalities are very similar to those observed in cells obtained from affected family members with the gray platelet syndrome (Fig. 3E), indicating that expression of GFI1 $\mathrm{B}^{\mathrm{Tr}}$ is sufficient to cause megakaryocytic abnormalities and that it functions in a dominant-negative manner.

\section{CELLULAR ANALYSES}

Studies involving Gfilb knockout mice have shown that Gfilb plays an important role in the development of several hematopoietic lineages. ${ }^{15,17,19}$ In humans, GFI1B is required for in vitro differentiation of erythrocytic and megakaryocytic lineages. ${ }^{18}$ We observed that megakaryocyte colonyforming cells were significantly more frequent in a bone marrow specimen from affected family members than in a specimen from a healthy person. In addition, the colonies from the affected family member were larger than those from a healthy control (Fig. 3A, and Fig. S7 in the Supplementary Appendix); this is in line with the increased number of megakaryocytes in the biopsy specimen from affected Family Member III.2 (Fig. 1I).

To determine whether GFI1B ${ }^{\operatorname{Tr}}$ was associated with other hematopoietic abnormalities, we performed immunophenotypic analyses of peripheral-blood specimens obtained from eight family members and five healthy controls. Myeloid and erythroid lineages were unaffected (data not shown). The surface expression of characteristic platelet markers, such as $\beta_{3}$ glycoprotein-CD36, $\alpha \mathrm{II} \beta$ integrin-CD41, and $\beta_{3}$ integrin-CD61, was also unperturbed in all tested persons. However, as compared with unaffected family members and healthy controls, five of six affected members had a marked decrease in the level of platelet surface-membrane glycoprotein $1 \mathrm{~b} \alpha-\mathrm{CD} 42 \mathrm{~B}$ within the $\alpha \mathrm{II} \beta$ integrin-CD41 platelet compartment (Fig. S8 in the Supplementary Appendix). In addition, strong expression of CD34, which is usually confined to immature hematopoietic progenitors, was detected on platelets from all affected family members in the study (Fig. 3B).
Because platelets are derived from megakaryocytes, ${ }^{1,2}$ we evaluated whether aberrant expression of surface molecules was also present on megakaryocytes. Immunostaining (with $\beta_{3}$ integrin-CD61) of a bone marrow-biopsy specimen obtained from affected Family Member III.2 confirmed the presence of megakaryocytes that had high levels of CD34 expression (Fig. 3C). Electron-microscopic analysis of the same biopsy specimens showed megakaryocytes with few alpha granules, which were small, irregularly shaped, and centrally located; the megakaryocytes were characterized by an extensive peripheral cytoplasm with irregular proplatelets, largely devoid of cell organelles (Fig. 3D, and Fig. S9 in the Supplementary Appendix). Consistently, bone marrow smears obtained from the same patient (Patient III.2) and her mother (Patient II.2) showed dysplastic megakaryocytes that were pale, as a result of diminished alpha granules (Fig. 3E). To determine whether these abnormalities were intrinsic to the cell, we stimulated CD34+ cells obtained from affected Family Members II.2 and III.2 to differentiate along the megakaryocytic lineage in culture. Megakaryocytic cells had dysplastic features such as those observed in the bone marrow aspirates (Fig. 3E). In addition, altered expression of $\mathrm{CD} 34$ and glycoprotein $1 \mathrm{~b} \alpha-\mathrm{CD} 42 \mathrm{~B}$ was observed in megakaryocytic cells that were positive for $\alpha \mathrm{II} \beta$ integrinCD41 (Fig. S10 in the Supplementary Appendix).

\section{DISCUSSION}

The gray platelet syndrome is a hereditary bleeding disorder characterized by platelets that lack alpha granules. ${ }^{5-10}$ Recently, mutations in NBEAL2 have been shown to cause an autosomal recessive form of the gray platelet syndrome. ${ }^{12-14} \mathrm{We}$ found that a mutation in GFI1B was causally related to autosomal dominant gray platelet syndrome.

In addition to large gray platelets that are almost devoid of alpha granules, other phenomena associated with the gray platelet syndrome, such as thrombocytopenia, emperipolesis, mild myelofibrosis, and low expression of platelet factor 4, were observed in affected persons. However, we also found new features of the gray platelet syndrome such as megakaryocytes and platelets that were strongly positive for the stemcell and progenitor-cell marker CD34, and had a 


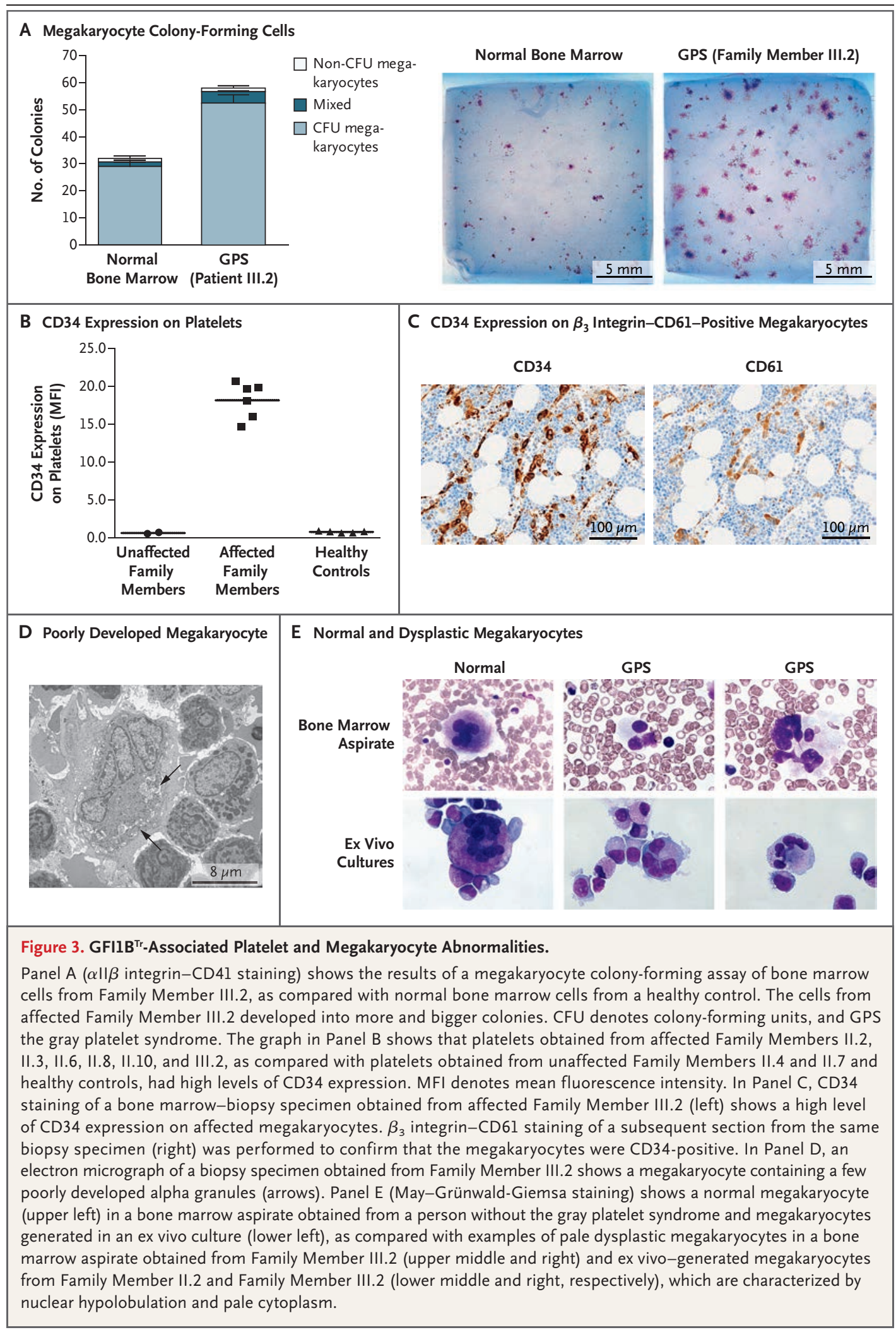

N ENGLJ MED 370;3 NEJM.ORG JANUARY 16, 2014

The New England Journal of Medicine 
diminished expression of glycoprotein $1 \mathrm{~b} \alpha-$ CD42B. Diminished glycoprotein $1 \mathrm{~b} \alpha-\mathrm{CD} 42 \mathrm{~B}$ platelet expression may contribute to the pathogenesis of the gray platelet syndrome, since glycoprotein $1 \mathrm{~b} \alpha-\mathrm{CD} 42 \mathrm{~B}$ plays an important role in the initial adhesion of platelets to vascular subendothelium after injury. Low platelet expression of glycoprotein $1 \mathrm{~b} \alpha-\mathrm{CD} 42 \mathrm{~B}$ may be related to the large platelets observed in affected persons, since large platelets are present in the Bernard-Soulier syndrome, a bleeding disorder caused by GP1BA/CD42B mutations. In addition, and possibly consequential to aberrant marker expression, we observed that megakaryocytes were abnormally distributed within the bone marrow, and they were frequently aligned in clusters along the sinuses in a stretched fashion. These features, in combination with the presence of morphologic and nuclear abnormalities in megakaryocytes, indicate that GFI $1 \mathrm{~B}^{\mathrm{Tr}}$ affects the terminal maturation of megakaryocytes.

GFI1B silencing and Gfilb ablation studies have shown that the protein is fundamental to the development of several blood-cell lineages. ${ }^{15,17-20}$ In the affected members of the family we studied, the presence of GFI1 ${ }^{\text {Tr }}$ was associated with major abnormalities only in the megakaryocytic lineage. The mutation in the gray platelet syndrome introduces a stop codon that results in a truncated GFI1B protein. The deleted region includes four amino acids that directly interact with DNA. Indeed, GFI1B ${ }^{\text {Tr }}$ expression did not inhibit gene expression, but it inhibited the function of nonmutant GFI1B. This indicates that the gray platelet syndrome may be caused not by GFI1B haploinsufficiency but rather by dominant-negative inhibition of the nonmutant protein. Indeed, forced GFI1B ${ }^{\operatorname{Tr}}$ expression in normal bone marrow cells resulted in severely dysplastic megakaryocytes that were similar to those observed in affected persons. These findings are consistent with mouse models showing that only complete Gfilb ablation disturbs in vitro development of megakaryocytes, whereas the loss of a single Gfilb allele is not sufficient to yield a clear phenotype. ${ }^{17}$ GFI1B ${ }^{\text {Tr }}$ retains several corepressor and transcription factor-interacting domains and may act in a dominant-negative manner by quenching proteins that would normally cooperate with nonmutant GFI1B. The identification of GFI1B target genes that may be disturbed by GFI1 $\mathrm{B}^{\mathrm{Tr}}$ would provide relevant insight into the molecular pathways that are key for megakaryopoiesis and platelet production.

In summary, our data indicate that GFI1B is a causative gene in autosomal dominant gray platelet syndrome. The truncated GFI1B mutant acts in a dominant-negative manner over the nonmutant protein and affects the normal development of megakaryocytes and platelets, reflecting the pivotal role of GFI1B in governing normal megakaryopoiesis and platelet production.

Disclosure forms provided by the authors are available with the full text of this article at NEJM.org.

We thank Dominique Duménil, Ph.D., for sharing a human GFI1B expression construct.
REFERENCES

1. Kaushansky K. Historical review: megakaryopoiesis and thrombopoiesis. Blood 2008;111:981-6.

2. Junt $T$, Schulze $H$, Chen $Z$, et al. Dynamic visualization of thrombopoiesis within bone marrow. Science 2007;317: 1767-70.

3. Rendu F, Brohard-Bohn B. The platelet release reaction: granules' constituents, secretion and functions. Platelets 2001; 12:261-73.

4. Maynard DM, Heijnen HF, Horne MK, White JG, Gahl WA. Proteomic analysis of platelet alpha-granules using mass spectrometry. J Thromb Haemost 2007;5:194555.

5. Raccuglia G. Gray platelet syndrome: a variety of qualitative platelet disorder. Am J Med 1971;51:818-28.

6. Nurden AT, Nurden P. The gray platelet syndrome: clinical spectrum of the disease. Blood Rev 2007;21:21-36.

7. White JG. Ultrastructural studies of the gray platelet syndrome. Am J Pathol 1979;95:445-62.

8. Gunay-Aygun M, Zivony-Elboum Y, Gumruk F, et al. Gray platelet syndrome: natural history of a large patient cohort and locus assignment to chromosome $3 \mathrm{p}$. Blood 2010;116:4990-5001.

9. Weiss HJ, Witte LD, Kaplan KL, et al. Heterogeneity in storage pool deficiency: studies on granule-bound substances in 18 patients including variants deficient in alpha-granules, platelet factor 4, betathromboglobulin, and platelet-derived growth factor. Blood 1979;54:1296-319. 10. Breton-Gorius J, Vainchenker W, Nurden A, Levy-Toledano S, Caen J. Defective alpha-granule production in megakaryocytes from gray platelet syndrome: ultrastructural studies of bone marrow cells and megakaryocytes growing in culture from blood precursors. Am J Pathol 1981;102:10-9.

11. Mori K, Suzuki S, Sugai K. Electron microscopic and functional studies on platelets in gray platelet syndrome. Tohoku J Exp Med 1984;143:261-87.

12. Gunay-Aygun M, Falik-Zaccai TC, Vilboux T, et al. NBEAL2 is mutated in gray platelet syndrome and is required for biogenesis of platelet $\alpha$-granules. Nat Genet 2011;43:732-4.

13. Kahr WH, Hinckley J, Li L, et al. Mutations in NBEAL2, encoding a BEACH protein, cause gray platelet syndrome. Nat Genet 2011;43:738-40.

14. Albers CA, Cvejic A, Favier R, et al. Exome sequencing identifies NBEAL2 as the causative gene for gray platelet syndrome. Nat Genet 2011;43:735-7.

15. van der Meer LT, Jansen JH, van der Reijden BA. Gfi1 and Gfi1b: key regulators of hematopoiesis. Leukemia 2010;24: 1834-43.

16. Zweidler-Mckay PA, Grimes HL, Flubacher MM, Tsichlis PN. Gfi-1 encodes a nuclear zinc finger protein that 
binds DNA and functions as a transcriptional repressor. Mol Cell Biol 1996;16: 4024-34.

17. Saleque S, Cameron S, Orkin SH. The zinc-finger proto-oncogene Gfi- $1 \mathrm{~b}$ is essential for development of the erythroid and megakaryocytic lineages. Genes Dev 2002;16:301-6.

18. Randrianarison-Huetz V, Laurent $B$, Bardet V, Blobe GC, Huetz F, Duménil D. Gfi-1B controls human erythroid and megakaryocytic differentiation by regulating TGF-beta signaling at the bipotent erythro-megakaryocytic progenitor stage. Blood 2010;115:2784-95.

19. Khandanpour C, Sharif-Askari E,
Vassen L, et al. Evidence that growth factor independence $1 \mathrm{~b}$ regulates dormancy and peripheral blood mobilization of hematopoietic stem cells. Blood 2010;116: 5149-61.

20. Person RE, Li FQ, Duan $Z$, et al. Mutations in proto-oncogene GFI1 cause human neutropenia and target ELA2. Nat Genet 2003;34:308-12.

21. Vassen L, Fiolka K, Möröy T. Gfi1b alters histone methylation at target gene promoters and sites of gamma-satellite containing heterochromatin. EMBO J 2006; 25:2409-19.

22. Lee S, Doddapaneni K, Hogue A, McGhee L, Meyers S, Wu Z. Solution struc- ture of Gfi-1 zinc domain bound to consensus DNA. J Mol Biol 2010;397:1055-66. 23. Saleque S, Kim J, Rooke HM, Orkin $\mathrm{SH}$. Epigenetic regulation of hematopoietic differentiation by Gfi- 1 and Gfi-1b is mediated by the cofactors CoREST and LSD1. Mol Cell 2007;27:562-72.

24. Kurstjens R, Bolt C, Vossen M, Haanen C. Familial thrombopathic thrombocytopenia. Br J Haematol 1968;15:305-17.

25. Vassen L, Fiolka K, Mahlmann S, Möröy T. Direct transcriptional repression of the genes encoding the zinc-finger proteins Gfi1b and Gfi1 by Gfi1b. Nucleic Acids Res 2005;33:987-98.

Copyright (c) 2013 Massachusetts Medical Society.

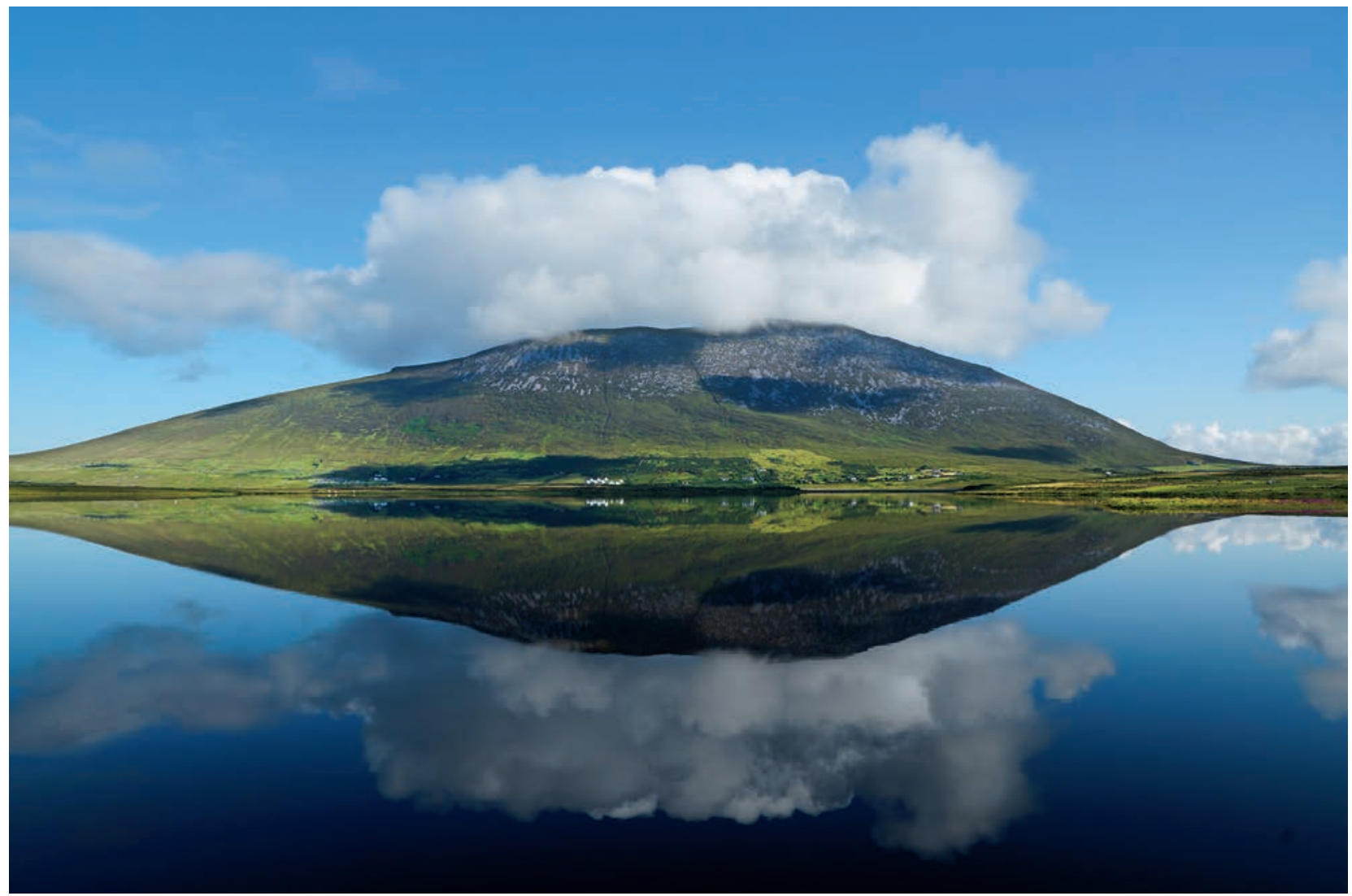

Keel Lough, Achill Island, Co. Mayo, Ireland

Charles Cowles, M.D. 